\title{
Validação transcultural da Escala de Avaliação de Limitações no Comportamento Social - SBS-BR
}

\author{
Lúcia Abelha Lima ${ }^{1}$ \\ Sylvia Gonçalves ${ }^{2}$ \\ Giovanni Lovisi ${ }^{3}$ \\ Basílio de Bragança Pereira ${ }^{4}$
}

Recebido: 13/8/2003 Aceito: 15/8/2003

\begin{abstract}
RESUMO
Este trabalho apresenta os resultados referentes à validação da versão brasileira da Social Behaviour Schedule - SBS, escala que avalia as limitações no comportamento social de pacientes psiquiátricos. O estudo de tradução e adaptação transcultural do instrumento foi realizado pelo Núcleo de Pesquisa do Instituto Municipal de Assistência à Saúde Juliano Moreira (IMASJM), RJ, e a coleta de dados foi feita nas Unidades Assistenciais do IMASJM. Análise das propriedades psicométricas da escala foi feita no Núcleo de Ensino e Saúde Coletiva (NESC) da UFRJ. A Escala de Avaliação do Comportamento Social foi submetida a uma backtranslation, assim como a um estudo piloto sendo avaliada por uma comissão de especialistas, para que fosse feita sua adaptação ao nosso contexto. A $S B S-B R$ mostrou-se uma escala com qualidades psicométricas de validade e fidedignidade satisfatórias, no que se refere à consistência interna da escala, bem como à sua validade discriminante e de construto. A análise da estrutura fatorial da escala nos permitiu a identificação de quatro síndromes comportamentais bem definidas. Estudos futuros deverão aprofundar a investigação das qualidades psicométricas da presente escala, no que se refere à sua confiabilidade com relação a interentrevistados e interentrevistadores. A Escala SBS-BR tem muito a contribuir na avaliação do impacto dos novos programas de saúde mental, principalmente no que diz respeito aos pacientes psiquiátricos com distúrbios severos e persistentes.
\end{abstract}

Unitermos: SBS; Validade; Limitações no comportamento social; Avaliação de serviços de saúde mental.

\section{ABSTRACT}

Transcultural validation of the Social Behaviour Schedule (SBS)

This article describes the psychometric properties of the Brazilian version of the Social Behaviour Schedule - SBS, developed to provide global assessments of functional living skills of chronically mentally ill individuals. Núcleo de Pesquisa do Instituto Municipal de Assistência à Saúde Juliano Moreira (IMASJM), RJ, has conducted the questionnaires translation and adaptation and the data collection took place in the health care units of IMASJM. Núcleo de Ensino e Saúde Coletiva (NESC) da UFRJ has conducted the analysis assessing the scale psychometric properties. The scale was submitted to a backtranslation, an evaluation by an expert committee and a pilot study in other to implement its adaptation to the Brazilian context. The results showed good psychometric properties of reliability and validity, in terms of the internal consistency of the scale, construct validity of the scale and its discriminant validity. Future studies should investigate inter-rater reability and inter-informant reliability. The SBSBR is an important instrument to evaluate mental health programs for severe mentally ill patients.

Keywords: SBS; Validity; Limitation in social behavior; Evaluation of mental health services.

\footnotetext{
Núcleo de Pesquisa/Centro de Estudos do Instituto Municipal de Assistência à Saúde Juliano Moreira - SMSRJ.

1 Pesquisadora do Núcleo de Pesquisa do Instituto de Assistência à Saúde Juliano Moreira - SMSRJ e Doutora em Saúde Pública pela ENSP/FIOCRUZ.

2 Pesquisadora do Núcleo de Pesquisa do IMASJM e Especialista em Saúde Mental da Escola Nacional de Saúde Pública/Fundação Oswaldo Cruz.

3 Professor de Psiquiatria da Universidade Federal do Estado do Rio de Janeiro.

4 Professor Titular NESC e COPPE da Universidade Federal do Rio de Janeiro.

Endereço para correspondência:

Lúcia Abelha Lima

Rua Projetada, D 330 F - Rio de Janeiro, RJ - CEP 22790-700

Tel.: (0xx21) 2498-3771

Fax: (0xx21) 2446-6673

E-mail:abelha@olimpo.com.br
} 


\section{Introdução}

A necessidade de avaliar os processos de reforma psiquiátrica objetivamente tem levado à implantação de projetos que utilizam escalas de avaliação para medir a resolutividade dos serviços. Isso é de fundamental importância para um planejamento adequado das futuras intervenções (O’Driscol, 1993).

O tratamento do paciente com transtorno mental grave representa um dos maiores desafios para as reformas no setor de saúde mental, porque requer cuidados especiais no processo de desinstitucionalização. Esses pacientes necessitam de apoio constante, além de avaliação do suporte social disponível. Precisam de serviços de reabilitação continuada em função de suas dificuldades para realização de atividades na vida diária e nas relações sociais.

A incapacidade nas doenças mentais está relacionada principalmente às dificuldades no comportamento, nas funções e nos papéis sociais do paciente. Os fatores sociais estão muito presentes no curso e no desenvolvimento dos transtornos mentais. Portanto, é importante que se utilizem instrumentos objetivos e confiáveis para avaliação do funcionamento social desses pacientes (Harvey, 1996).

Em função de sua variabilidade, o diagnóstico psiquiátrico não é considerado um instrumento suficiente para prever os resultados da reabilitação. Aferir o grau de desempenho psicossocial é fundamental para o planejamento e a avaliação do desempenho dos pacientes na comunidade e estruturar programas de reabilitação psicossocial (Wykes e Sturt, 1987; Wing, 1989; Wiersma, 1996; Gonçalves et al., 2001).

Muitas escalas têm sido utilizadas para medir as dificuldades no comportamento social do paciente psiquiátrico grave. Entre elas, a Social Behaviour Schedule (SBS) tem se mostrado um instrumento adequado, principalmente por ser uma das mais bem conhecidas e mais utilizadas internacionalmente e por se prestar bem à avaliação de pacientes mais comprometidos, que têm dificuldades para responder às questões propostas, pois estas são fornecidas por um informante que conhece bem o paciente.

No Brasil, a quantidade de escalas validadas ainda é pequena, principalmente no que diz respeito a instrumentos para medir as dificuldades no comportamento social. A validação e a adaptação cultural desses instrumentos à nossa realidade é importante não só para a comparação com estudos de outros países, mas principalmente para o planejamento e monitoramento dos pacientes em seu convívio na comunidade e na atenção individualizada nos programas de reabilitação psicossocial.
O objetivo desse estudo é realizar a validação transcultural da escala Social Behaviour Schedule (SBS) para a avaliação do comportamento social em uma população de pacientes psiquiátricos de longa permanência.

\section{Metodologia}

Esta pesquisa envolveu duas etapas. A primeira etapa da pesquisa incluiu a tradução e adaptação das questões da escala para o contexto brasileiro e um estudo piloto visando ajustar a formulação das questões à população-alvo. A segunda etapa envolveu a análise estatística das propriedades psicométrica e fatorial da versão brasileira da escala.

\section{Local da pesquisa}

A pesquisa foi desenvolvida no Instituto Municipal de Assistência à Saúde Juliano Moreira (IMASJM), localizado no Rio de Janeiro. O IMASJM abriga atualmente pacientes de ambos os sexos em seis diferentes unidades, tais como: Núcleo Franco da Rocha (NFR) e Núcleo Teixeira Brandão (NTB) femininos; Núcleo Ulisses Viana (NUV), Núcleo Rodrigues Caldas (NRC) e Pavilhão Agrícola (PA) masculinos e Centro de Reabilitação e Integração Social (CRIS) - feminino e masculino. A maior parte da clientela não possui vínculo familiar, não recebe visitas e apresenta participação social limitada.

Atualmente a instituição passa por uma reformulação de seu modelo de assistência, com a criação de Residências Terapêuticas e Lares de Acolhimento para pacientes com diferentes níveis de autonomia, dentro e fora dos limites da antiga Colônia Juliano Moreira.

\section{População estudada}

Para o estudo de validação transcultural foram utilizados dados de todos os pacientes do IMASJM. Essa população é constituída de 881 pacientes $(58,6 \%$ femininos e $41,4 \%$ masculinos), com idades que variam de 24 a 98 anos, sendo a média de idade de 65,8 anos $(\mathrm{dp}=11)$ e o tempo médio de internação de 37,3 anos $(\mathrm{dp}=11,5)$. O nível de escolaridade é baixo, com 337 (38,3\%) pacientes analfabetos e $199(22,6 \%)$ apenas alfabetizados. A maior parte da clientela não recebe visitas (72\%) e não possui vínculo empregatício, embora 43,4\% recebam uma bolsaauxílio do IMASJM ou pensão da previdência social.

Quanto ao perfil diagnóstico: 63\% têm diagnóstico de esquizofrenia, transtornos esquizotípicos ou transtornos delirantes; $19 \%$ têm retardo mental; $6 \%$ têm epilepsia e $12 \%$ têm outros diagnósticos. 


\section{A escala original}

A Social Behaviour Schedule (SBS) foi desenvolvida para medir o comportamento social de pacientes de longa permanência tanto em enfermarias como em residências na comunidade. É composta de 21 itens, a maioria pontuados de $0 \mathrm{a} 4$. Os 20 primeiros itens são bastante específicos e o último item permite ao informante incluir problemas diversos, como alcoolismo e distúrbios alimentares, mas não foram freqüentes o suficiente, em estudos anteriores, para justificar um item em separado. As informações são obtidas a partir das observações de um informante, sobre o comportamento do paciente no último mês. Foi especificamente desenhada para refletir as dificuldades no comportamento social (Wykes e Sturt, 1986).

$\mathrm{Na}$ avaliação dos 21 itens da SBS, o método de pontuação BSM (Mild and Severe Behaviour Problems Score) e BSS (Severe Behaviour Problems Scores) foram utilizados para determinar a presença ou ausência de problemas de comportamento social. De acordo com o método BSM, o problema é considerado presente quando a pontuação das respostas é 2,3 ou 4 e ausente quando essa pontuação é 0 ou 1.

Método de pontuação BSM:

0 ou $1=0$ [distúrbio de comportamento leve

(1) ou inexistente (0)]

2,3 ou $4=1$ [distúrbio de comportamento

moderado (2) ou severo $(3,4)]$

De acordo com o método BSS, o problema é ausente quando a pontuação é 0 , considerado leve quando a pontuação das respostas é 2 e considerado severo quando a pontuação é 3 ou 4 .

Método pontuação BSS:

$0=$ distúrbio de comportamento inexistente

2 = distúrbio de comportamento moderado

3 e 4 = distúrbio de comportamento severo

No presente estudo utilizamos para as análises a pontuação BSM.

A escala original na língua inglesa apresenta propriedades psicométricas adequadas de consistência interna, uma vez que os coeficientes alfa de Cronbach variaram de 0,71 a 0,75 para os escores BSS e BSM nas diversas análises de consistência interna.

Etapa I: tradução, retrotradução e adaptação para o contexto brasileiro

A adaptação transcultural da presente escala para a nossa realidade cultural foi realizada segundo os procedimentos recomendados pela OMS (1996) e por Vallerand (1989) para a adaptação transcultural de instrumentos de medidas. Este procedimento consta das seguintes etapas: 1. tradução; 2. revisão da tradução por um grupo bilíngüe; 3. retrotradução; 4 . avaliação da retrotradução; 5. estudo piloto I; 6. revisão das questões a partir do estudo piloto.

A tradução da escala já havia sido feita por Caetano (1997) e foi revisada por três psiquiatras. A retrotradução foi feita por um epidemiologista bilíngüe e a avaliação da retrotradução por um grupo de profissionais bilíngues com experiência na área. Nenhum item foi suprimido na discussão de avaliação da retrotradução da escala (Anexo I).

\section{Estudo piloto}

O estudo piloto visou a ajustar a formulação das questões da versão preliminar da SBS-BR, em função da população-alvo na qual a escala seria aplicada, a fim de se assegurar uma boa compreensão e precisão do texto.

Foi realizado em uma amostra de 23 mulheres do Núcleo Franco da Rocha. As entrevistas foram realizadas por uma psiquiatra e uma psicóloga que participaram dos grupos de revisão da tradução e avaliação da retrotradução da escala. Foram entrevistados técnicos das unidades que conheciam bem o convívio social dos pacientes.

A partir do estudo piloto, foram realizados painéis de discussão com representantes dos técnicos das unidades em que foram debatidas modificações na redação e em alguns termos da escala que facilitassem sua compreensão. Foram feitas pequenas modificações na formulação das questões. Não foi sugerida a eliminação de nenhuma das questões do instrumento.

\section{da escala \\ Etapa II: estudo das propriedades psicométricas}

Nesta segunda etapa da pesquisa, a aplicação da versão adaptada da escala foi feita em toda a população $(\mathrm{n}=881)$ do Instituto Municipal de Assistência à Saúde Juliano Moreira (IMASJM), com o objetivo de verificar suas propriedades psicométricas e fazer a análise fatorial.

O procedimento de aplicação da escala foi iniciado após o projeto ter sido aprovado pela comissão de ética em pesquisa do IMASJM, garantindo-se o consentimento, o sigilo e o anonimato das respostas. A aplicação da escala foi realizada no período de um mês por dez pesquisadores graduados em psicologia, contratados pela Secretaria Municipal de Saúde para essa atividade e treinados por uma psiquiatra e uma psicóloga, co-autoras desse estudo. O treinamento teve a duração de 12 horas e foi constituído de três módulos: 1. análise e discussão da escala; 2. aplicação piloto da escala e 3. painéis de discussão.

Os entrevistadores foram alocados nas seis unidades do IMASJM e supervisionados pela mesma equipe de treinamento. Em cada unidade, as entrevistas foram 
realizadas com os técnicos da unidade mais próximos aos pacientes. As instruções para resposta e cada um dos itens foi lido pelo entrevistador para cada respondente. As respostas foram anotadas pelo entrevistador no próprio questionário.

As análises foram realizadas utilizando o programa SPSS 10.1 disponível no Núcleo de Pesquisa do IMASJM.

\section{Resultados}

\section{Frequência de limitações no comportamento social}

A população do IMASJM apresentou maiores dificuldades nas áreas da aparência pessoal e higiene $(50,6 \%)$, comunicação $(46 \%)$, concentração $(41,1 \%)$ e inatividade $(37,1 \%)$. Um percentual muito pequeno da população apresentou problemas em relação a comportamento suicida e destrutivo, agressividade, ataques de pânico e fobias, realização de idéias bizarras e comportamento social inadequado. O número médio de problemas moderados e graves (escore BSM) foi 3,9 sd 3,1 (mediana $=3$; máximo $=17$ ). Esses resultados são esperados, por se tratar de uma clientela com longos anos de internação, que mantém poucos laços fora da instituição, desprovida de lazer e, em sua maioria, não mantém contato com a família. Comportamento sexual inadequado, depressivo e hostil aparece em pequeno percentual na população (Tabela 1).

\section{Análise fatorial}

A análise fatorial foi realizada com as 14 variáveis que obtiveram na matriz de correlação um coeficiente maior que 0,3 . Foi utilizado o método dos componentes principais, após rotação varimax. Foram obtidos quatro fatores que explicam $57,8 \%$ da variação dos dados.

$\mathrm{Na}$ tabela de cargas fatoriais para uma solução com quatro fatores e rotação varimax, utilizamos apenas valores maiores do que 0,4 (valor absoluto).

Nomeamos os quatro fatores da seguinte forma, procurando refletir os itens de cada um deles:

- afastamento social: var 1 , var 17 , var 18 , var 19, var 20;

- comportamento social embaraçoso: var 2, var 3, var 4, var 10, var 13;

- comportamento inquieto: var 9, var 12;

- comportamento de natureza hostil: var 5, var 14.

As demais variáveis, embora tenham permanecido na escala, não foram consideradas na análise fatorial pelas razões estatísticas descritas anteriormente.

Tabela 1 Distribuição das limitações do comportamento social* aferidas pela SBS nos pacientes do IMASJM

\begin{tabular}{|c|c|c|}
\hline Tipo de problema & Número & $\%$ \\
\hline Comunicação: tomando a iniciativa & 405 & 46,0 \\
\hline Conversação: incoerência & 221 & 25,1 \\
\hline Conversação: excentricidade/inapropriação & 107 & 12,1 \\
\hline Interação social: & 210 & 23,8 \\
\hline $\begin{array}{l}\text { Interação social: proporção de contatos sociais que são por } \\
\text { natureza hostis }\end{array}$ & 91 & 10,3 \\
\hline Interação social: comportamento chamativo & 82 & 9,3 \\
\hline Idéias ou comportamento suicida ou de auto-agressão & 6 & 0,7 \\
\hline Ataques de pânico e fobias & 60 & 6,8 \\
\hline Inquietação e hiperatividade & 181 & 20,6 \\
\hline Rir e falar sozinho & 203 & 23 \\
\hline Realização de idéias bizarras & 28 & 3,2 \\
\hline Estereotipias e maneirismos & 132 & 15 \\
\hline Hábitos ou maneiras socialmente inaceitáveis & 159 & 18 \\
\hline Comportamento destrutivo & 21 & 2,4 \\
\hline Depressão & 55 & 6,2 \\
\hline Comportamento sexual inadequado & 34 & 3,9 \\
\hline Aparência pessoal e higiene & 446 & 50,6 \\
\hline Lentidão & 152 & 17,3 \\
\hline Inatividade & 327 & 37,1 \\
\hline Concentração & 362 & 41,1 \\
\hline $\begin{array}{l}\text { Comportamento não especificado em outro lugar que } \\
\text { impeça progressos }\end{array}$ & 174 & 19,8 \\
\hline
\end{tabular}

* Pontuação 2 ou mais nos 21 itens da SBS 
Tabela 2 Cargas fatoriais para uma solução com quatro fatores com rotação varimax a partir de uma análise de componentes principais aplicada aos itens da escala SBS-BR

\begin{tabular}{lrccc}
\hline \multicolumn{5}{c}{ Componentes } \\
\hline & 1 & 2 & 3 & 4 \\
SBS1a & 753 & & & \\
SBS2a & & 806 & & \\
SBS3a & & 748 & & \\
SBS4a & 421 & 529 & & \\
SBS5a & & & 766 & \\
SBS9a & & & & \\
SBS10a & & 566 & & \\
SBS12a & & & & \\
SBS13a & & 456 & & \\
SBS14a & & & \\
SBS17a & 656 & & \\
SBS18a & 667 & & \\
SBS19a & 788 & & \\
SBS20a & 718 & & \\
\hline
\end{tabular}

\section{Validade de construção: consistência interna}

A fidedignidade da presente escala foi avaliada a partir da análise da consistência interna dos 21 itens da escala. O valor para o coeficiente alfa de Cronbach foi 0,779, o que indica uma boa homogeneidade e consistência dos itens, pois esses valores se situaram acima do critério estabelecido (critério mínimo $=0,70$ ) para escalas contendo mais de dez itens, tal como sugerido por Gulliksen (1950) e Martinez (1995).

$\mathrm{Na}$ análise estatística de consistência interna, as correlações item-total de três variáveis (var 7, var 15 e var 16) se situaram abaixo do critério mínimo requerido $(\mathrm{r}=0,20)$ para uma consistência interna adequada. No entanto, essas variáveis não foram excluídas da escala pelas seguintes razões:

1. São variáveis que se referem a comportamentos mais raros em pacientes institucionalizados a longo tempo (idéias suicidas, depressão e comportamento sexual inadequado), mas são relevantes para pacientes que vivem na comunidade.

2. Na análise estatística de consistência interna separada para as diversas unidades do IMASJM, em algumas unidades essas variáveis apresentam correlação item-total acima do critério mínimo requerido.

\section{Validade discriminante}

A validade discriminante da escala SBS-BR foi verificada comparando-se os escores obtidos pelos pacientes das seis diferentes unidades existentes no instituto psiquiátrico estudado. Uma vez que essas unidades abrigam grupos de pacientes que diferem em termos de grau de limitações no comportamento social, a presente escala deveria ser sensível para detectar diferenças entre eles, em termos de suas limitações de conviver socialmente, o que atestaria a validade discriminante da SBS-BR. Essa comparação foi feita por meio da análise de variância (ANOVA). Os resultados indicaram que os grupos de pacientes das seis unidades do instituto apresentaram diferenças estatisticamente significativas entre elas no escore total $(\mathrm{F}=11.447 ; \mathrm{p}<0,000)$.

Verificamos também a análise discriminante da escala em relação aos quatro fatores (afastamento social, comportamento social embaraçoso, comportamento inquieto e comportamento de natureza hostil) por meio da análise de variância (ANOVA), o que é possível já que os fatores são ortogonais ou não correlacionados.

\section{Afastamento social}

A análise de variância (ANOVA) em relação ao primeiro fator $(\mathrm{F}=16,38 ; \mathrm{p}<0,000)$ indica uma diferença significativa no escore do SBS nas diferentes unidades do IMASJM.

Ao observarmos a figura 1, vemos que o CRIS, NFR e PA apresentam os menores escores nesse fator, enquanto o NRC e o NTB têm as maiores médias, o que é coerente com as características dos pacientes desses núcleos.

\section{Comportamento social embaraçoso}

O segundo fator apresenta uma análise de variância $\mathrm{F}=3,839(\mathrm{p}<0,005)$, o que também indica uma diferença significativa entre os escores da SBS nas diversas unidades do IMASJM, sendo que as maiores médias aparecem nas unidades NRC e PA. Entretanto só observamos diferenças significativas entre o NRC e as unidades NUV e NFR (Figura 1).

\section{Comportamento inquieto}

A análise de variância (ANOVA) em relação ao terceiro fator (2,002 não-significativo) indica uma diferença significativa entre os escores do SBS nas diferentes unidades do IMASJM. As maiores médias são das unidades NFR e NTB. As unidades do IMASJM não diferem significativamente em relação a esses sintomas (Figura 1).

\section{Comportamento de natureza hostil}

O quarto fator apresenta uma análise de variância $\mathrm{F}=5,548(\mathrm{p}<0,000)$, o que indica uma diferença significativa no escore do SBS nas diferentes unidades do IMASJM. O CRIS apresenta maior média neste fator. 
As diferenças significativas aparecem entre o CRIS e as unidades NFR, NTB e NUV. O comportamento de natureza hostil não discrimina as demais unidades entre si nem foram encontradas diferenças significativas entre o CRIS e o PA e o NRC (Figura 1).

\section{Discussão}

Existe uma grande necessidade de instrumentos padronizados que sejam capazes de medir a habilidade social de pacientes de longa permanência, assim como as mudanças nesse comportamento ao logo do tempo. A SBS adaptada para o contexto brasileiro mostrou-se um instrumento adequado para essa finalidade.

A escala apresentou qualidades psicométricas de validade e fidedignidade satisfatórias no que se refere à consistência interna, assim como à sua validade discriminante e validade de construto. Sua elevada consistência interna $(\alpha=0,779)$, acima do critério requerido, atesta sua fidedignidade. $\mathrm{O}$ coeficiente de alfa de Cronbach para o escore BSM foi de 0,766 , muito próximo ao obtido para a versão original da SBS, 0,75 (Wykes e Sturt, 1986).

Em virtude desses índices adequados, todos os 21 itens da escala original foram mantidos na versão brasileira, que poderá, portanto, avaliar as limitações no comportamento social dos pacientes nas mesmas áreas da versão original.

O estudo da estrutura fatorial da escala nos permitiu a identificação de quatro síndromes comportamentais bem definidas. A população do IMASJM apresenta altos percentuais de problemas em três itens $(1,19,20)$ do fator afastamento social.

Harvey et al. (1996) realizou um estudo sobre a capacidade da SBS em discriminar síndromes em pacientes esquizofrênicos. Fez uma análise fatorial em que foram excluídos os itens da escala que tiveram um coeficiente de correlação de Pearson menor ou igual a 0,4 e a partir dos 12 itens restantes encontrou quatro fatores:

- distúrbio de pensamento (fator 1): var 2, var 3 , var 20;

- afastamento social (fator 2): var 1, var 17, var 18 , var 19 ;

- humor depressivo (fator 3): var 7, var 15;

- comportamento anti-social (fator 4): var 5, var 13 , var 14 .

O estudo de Harvey (1996) também encontrou altos escores no fator afastamento social em pacientes de longa permanência, o que foi confirmado no estudo atual. A distribuição das variáveis nos fatores, no estudo

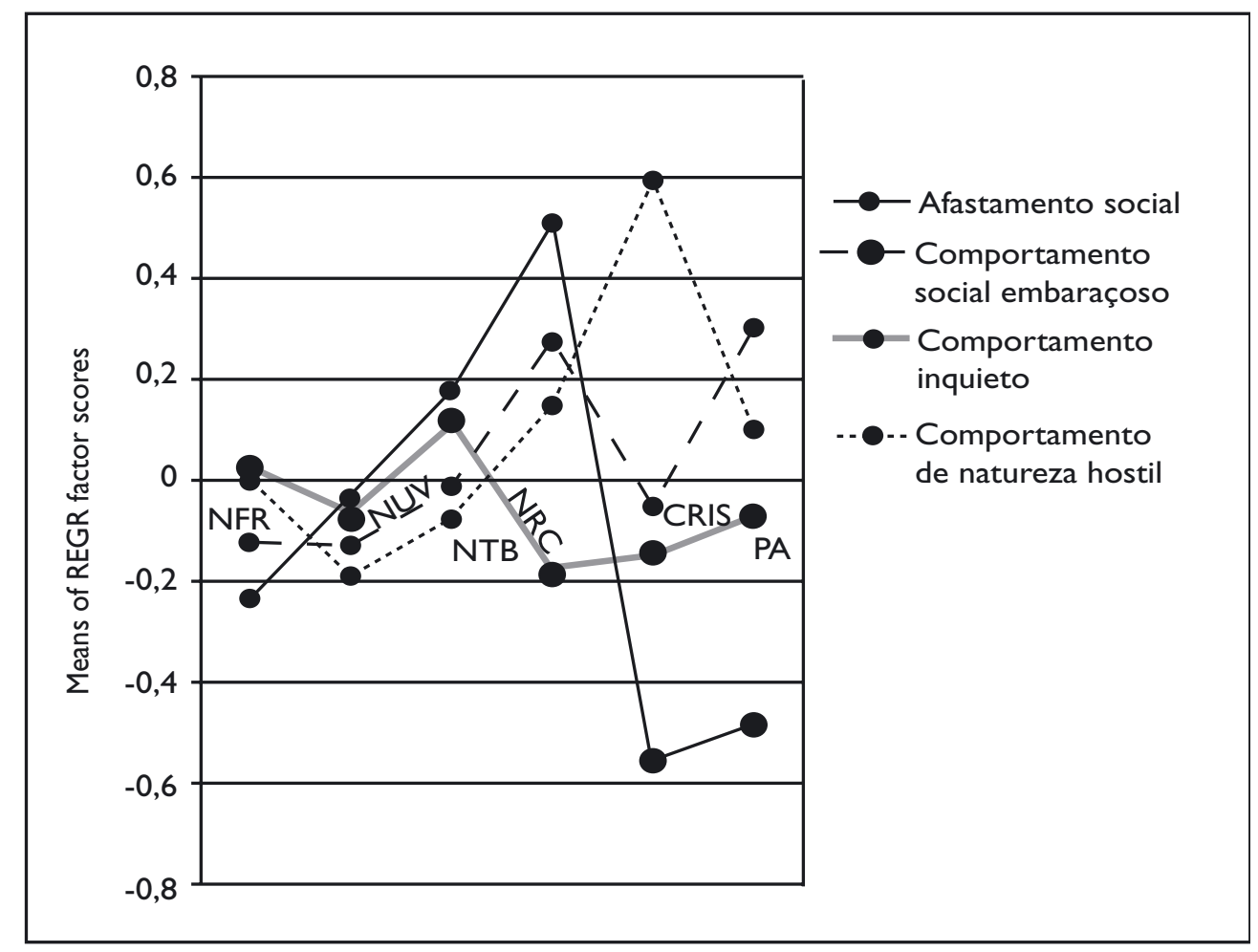

Figura 1 Análise de variância (ANOVA) relativa aos quatro fatores (afastamento social, comportamento social embaraçoso, comportamento inquieto e comportamento de natureza hostil) da SBS-BR entre as seis unidades do IMASJM. 
atual, foi bastante semelhante ao estudo de Harvey, principalmente no que diz respeito aos sintomas negativos (fator afastamento social - variáveis 1, 17, 18, 19 e 20).

Quanto à validade discriminante, verificamos que, na comparação das unidades de acordo com cada um dos fatores, encontramos diferenças significativas. As unidades do IMASJM têm características diferentes no que diz respeito a autonomia, sexo e convívio social dos pacientes. Entre as unidades, o CRIS, responsável pelos pacientes que vivem na comunidade, destaca-se por apresentar a menor média de pontuação no fator Afastamento Social. Esses pacientes são mais jovens, têm maior autonomia e foram selecionados entre os pacientes do IMASJM e do Hospital Jurandir Manfredini (hospital de pacientes agudos) para morarem nas residências terapêuticas. Os núcleos que apresentaram maiores médias neste fator foram os das unidades NTB e NRC, que abrigam os pacientes mais idosos e com maior comprometimento na iniciativa e na atividade espontânea.

As unidades que obtiveram as maiores médias no fator comportamento Social Embaraçoso foram o PA e o $\mathrm{NRC}$, núcleos masculinos. O fator Comportamento Inquieto não apresentou diferenças significativas entre os núcleos, e é um fator que tem como variáveis a hiperatividade e maneirismos que se distribuem de maneira semelhante em todos os núcleos. O CRIS apresentou a maior média em relação ao fator Comportamento de Natureza Hostil, talvez por ser uma unidade de pacientes mais jovens e mais submetidos a estímulos do mundo exterior. Os demais núcleos não apresentaram diferença entre si em relação a esse fator. Os resultados positivos obtidos para a validade discriminante dessa escala indicam também que ela é sensível para discriminar grupos distintos de pacientes psiquiátricos.

A aplicação da Social Behavioral Schedule mostrou-se um relevante instrumento no fornecimento de informações sobre a avaliação de serviços de saúde mental na Inglaterra, projeto TAPS (Leff e Trieman, 2000; Trieman e Leff, 2002), e a SBS-BR poderá representar a mesma utilidade no Brasil.
A adaptação transcultural da SBS-BR seguiu as recomendações estabelecidas na literatura para esse tipo de procedimento, por meio da participação de um grupo de especialistas e da aplicação de um estudo piloto na população-alvo. Isso permitiu uma formulaçao clara dos itens e uma equivalência com a versão original, adequando a escala à realidade brasileira. Estudos futuros deverão aprofundar a investigação das qualidades psicométricas da presente escala no que se refere à sua confiabilidade interentrevistadores e interentrevistados.

A escala SBS-BR é um instrumento importante para auxiliar no planejamento e na avaliação de programas que se relacionam à saída dos pacientes do hospital para a comunidade. A avaliação dos pacientes com essa escala poderá fornecer informações sobre suas limitações no comportamento social, informações essas que ajudarão a planejar os suportes sociais necessários para sua reinserção na comunidade, assim como os aspectos da sua convivência social poderão ser melhor trabalhados.

Além do mais, a utilização da escala também poderá ser útil para monitorar mudanças no comportamento social dos pacientes, com uso de medicações, em ensaios clínicos, uma vez que mede diferentes aspectos das limitações sociais apresentadas por eles.

A avaliação contínua dos serviços de saúde mental vem sendo estimulada pela Organização Mundial de Saúde. Entre os diversos aspectos que envolvem os transtornos psiquiátricos, certamente as limitações sociais estão entre os mais relevantes, pois são muitas vezes impeditivas da adaptação aos serviços e do convívio com a comunidade. Para organizar os programas de forma confiável, é indispensável a disponibilidade de instrumentos que tenham tido suas propriedades psicométricas estudadas e sido validados para nossa cultura de acordo com procedimentos padronizados.

Certamente a SBS-BR tem muito a contribuir na avaliação do impacto dos novos programas de saúde mental, principalmente no que diz respeito aos pacientes psiquiátricos com distúrbios severos e persistentes. 